\title{
Evaluation of Herbicides for Control of Weeds in Wheat (Triticum aestivum L.)
}

\author{
Deepesh Jaiswal $^{1}$, M. Devender Reddy ${ }^{2 *}$, Girish Pandey ${ }^{3}$ and Anuj Kumar ${ }^{4}$ \\ ${ }^{1}$ School of Agriculture, ITM University, Gwalior - 474001, (M.P.), India \\ ${ }^{2}$ M.S. Swaminathan School of Agriculture, Centurion University of Technology and \\ Management, Paralakhemundi, Odisha -761211, India \\ *Corresponding author
}

\section{A B S T R A C T}

\section{Keywords}

Post emergence herbicides, Weed control index, Weed control efficiency, Wheat

\section{Article Info}

Accepted:

05 April 2020

Available Online:

10 May 2020
A field experiment on evaluation of herbicides for control of weeds in wheat (Triticum aestivum L.) was conducted at School of Agriculture, ITM University, Gwalior, Madhya Pradesh during the winter season of 2015-16. The experiment comprised of ten post emergence herbicides application along with weed free and weedy check treatments. Maximum weed control efficiency was observed in weed free treatment followed by Sulfosulfuron + Metsulfuron, Isoproturon and Sulfosulfuron treatments. Sulfosulfuron + Metsulfuron recorded lowest weed index. The maximum grain yield was observed in weed free which was statistically at par with that observed with application of Sulfosulfuron + Metsulfuron, Isoproturon, and Sulfosulfuron. Highest straw yield was recorded in Sulfosulfuron which was statistically at par with weed free, Sulfosulfuron+ Metsulfuron, Mesosulfuron + Iodosulfuron, Isoproturon, Pinoxaden + Metsulfuron and Pinoxaden + 2,4D-E applied treatments. The lower grain and straw yield was recorded under weedy check which was significantly inferior to rest of the treatments. The harvest index was in the range of 40.80 to $47.02 \%$ under different weed control treatments. The results indicate that application of Sulfosulfuron + metsulfuron $\left(30+2 \mathrm{~g} \mathrm{ha}^{-1}\right)$ or isoproturon $\left(1000 \mathrm{~g} \mathrm{ha}^{-1}\right)$ are most remunerative and effective herbicides for weed management in irrigated wheat under sandy loam soils of Northern Madhya Pradesh.

\section{Introduction}

Wheat is one of the most important rabi cereals contributing $35 \%$ of total food grain production in our country. Heavy infestation of weeds alone causes $33 \%$ reduction in yield of wheat. Wheat crop gets infested with heavy population of Phalaris minor Retz., Avena ludoviciana Dur., Chenopodium album L., Melilotus indica All., Coronopus didymus L.,
Rumex retroflexus L., Vida sativa L. and Anagallis arvensis L.

The critical period of weed control in wheat is 30-45 days after sowing and crop should be kept weed free during this period. For the control of complex weed flora (grass and broadleaf weeds) and to provide long term residual weed control, application of different combinations of herbicides is needed. Tank 
mix combinations or ready mixtures are advantageous over sequential application due to saving in application timing and cost. The effectiveness of grass herbicides are generally reduced when mixed with broad-leaved herbicides (Damalas and Eleftherohorinos, 2001). Synergism/ compatibility have been found to occur more frequently in mixtures where the companion herbicides belong to the same chemical groups (Damalas, 2004). Sulfosulfuron + Metsulfuron are compatible (Chhokar et al., 2007) but tank mix application of grass herbicides (Clodinafop, Fenoxaprop, Tralkoxydim and Pinoxaden) with either 2, 4 - D or Metsulfuron is antagonistic (Mathiassen and Kudsk, 1998). To avoid antagonism, the grassy and broadleaved herbicides should be applied sequentially.

For controlling broadleaved weeds along with grasses, application of Isoproturon in combination of 2,4-D, Sulfosulfuron and Metsulfuron-methyl (MSM) are recommended (Pandey et. al., 2006, Singh and Singh, 2002).

The application of isoproturon + 2,4-D at 1.0 $+0.5 \mathrm{~kg} / \mathrm{ha}$ produced significantly higher grain yield (Kumara et al., 2019). Metribuzin has been found effective against associated weeds of wheat (Dixit and Bhan, 1997). Continuous use of Isoproturon led to the development of evolutionary resistant biotype and shift in weed flora (Malik and Singh, 1995).

A number of herbicides are, therefore, necessary to be evaluated for controlling weeds from the point of eco-safety and cost effective as the manual weeding or through animal drawn are costly. Keeping the above points in view, an experiment was conducted to evaluate herbicides for control of weeds in wheat (Triticum aestivum L) during rabi season of 2015-16.

\section{Materials and Methods}

An experiment was conducted to evaluate post emergence herbicides for control of weeds in wheat (Triticum aestivam L.) at ITM University, Gwalior, Madhya Pradesh during rabi season of 2015-16. The experiment site falls under humid sub-tropical climate and located in between $23^{\circ} 10^{\prime} \mathrm{N}$ latitude and $79^{\circ}$ $54^{\prime}$ E longitudes at an elevation of 411.98 meters above mean sea level. The soil type of experimental field was sandy loam in nature with $\mathrm{pH}$ of 7.4 and EC $0.29 \mathrm{dsm}^{-1}$, having 242 $\mathrm{kg}$ available nitrogen, $20.5 \mathrm{~kg}$ available phosphorus, $456 \mathrm{~kg}$ available potassium, 8.1 $\mathrm{kg}$ available sulphur per hectare.

During the crop growth period, the maximum temperature varied between $18.9{ }^{\circ} \mathrm{C}$ in January third week to $40.1{ }^{\circ} \mathrm{C}$ in April first week and minimum temperature ranged from $3.9{ }^{\circ} \mathrm{C}$ in third week of December to $23{ }^{\circ} \mathrm{C}$ in second week of April.

The experiment comprised of ten treatments of post emergence herbicides and their combinations along with hand weeding and no hand weeding (control) (Table 1). The experiment was laid out in Randomized block design with three replications.

A pre sowing irrigation was given to the experimental field and when it came in the condition, two cross ploughings followed by planking was done. The plots were prepared by forming channels and bunds and leveled.

Wheat variety GW-322 was sown by using seed rate of $100 \mathrm{~kg} \mathrm{ha}^{-1}$ on 29 November 2015 with funnel attached desi plough by maintaining row-to-row distance of $20 \mathrm{~cm}$. The crop was harvested on April 26, 2016. First weeding was done at 20 days after sowing and second at 35 days after sowing in hand weeded plot. The quantity of herbicides as per treatments was sprayed by knap - sack 
sprayer with flat fan nozzle and the quantity of water used was $6001 \mathrm{ha}^{-1}$.

The nutrients were applied at $120 \mathrm{~kg} \mathrm{~N}, 60 \mathrm{~kg}$ $\mathrm{P}_{2} \mathrm{O}_{5}$ and $40 \mathrm{~kg} \mathrm{~K}_{2} \mathrm{O} \mathrm{ha}^{-1}$. The full dose of $\mathrm{P}_{2} \mathrm{O}_{5}$ and $\mathrm{K}_{2} \mathrm{O}$ and half dose of nitrogen were drilled at $8 \mathrm{~cm}$ deep in the field at the time of sowing as a basal dose. Remaining half dose of nitrogen was applied in two equal splits after first and second irrigation. The nitrogen was applied through urea, $\mathrm{P}_{2} \mathrm{O}_{5}$ through Single Super Phosphate and $\mathrm{K}_{2} \mathrm{O}$ through Mureate of Potash. All the agronomic management practices were done uniformly in all the treatments. Six irrigations were given during the entire period of crop, besides pre sowing irrigation. The observations on weed population and weed dry weight were recorded at 30 DAS and harvest and the weed control efficiency, weed index were estimated by using the formulae as given below.

\section{Weed control efficiency (\%)}

Weed control efficiency of the various treatments were worked out with the help of the following formula.

Weed control efficiency $(\%)=\frac{X-Y}{X} \times 100$

Where,

$\mathrm{X}=$ Dry matter production of weeds in unweeded plot.

$\mathrm{Y}=$ Dry matter production of weeds in treated plot.

\section{Weed index $(\%)$}

The weed index (WI) was calculated by using the following formula (Gill and Vijay Kumar, 1969).

$$
\text { W.I. }=\frac{X-Y}{X} \times 100
$$

Where, $\mathrm{X}=$ Yield from maximum weed free plot.

$\mathrm{Y}=$ Yield from other treated plot.
The data on plant height was recorded on five plants which were tagged randomly in each treatment from each replication. The observations on number of tiller per meter row length and yield attributes number of effective tiller per meter row length, ear head length $(\mathrm{cm})$, number of grains per ear head, 1000 grain weight and biological, grain and straw yield were recorded.

The data obtained on various observations were subjected to statistical analysis by using the techniques of the analysis of variance (ANOVA) and the treatment was tested by $\mathrm{F}$ test and Critical difference (CD) at 5\% level of significance (Panse and Sukhatme, 1989) for each character to compare the differences among treatment means.

\section{Results and Discussion}

\section{Weeds}

\section{Weed flora}

Major monocot weeds were Cyperus rotundus, Phalaris minor and Asphodelus tenuifolius and major dicot weeds were Chenopodium album, Anagalis arvensis, Convolvulus arvensis and Medicago hispida. Out of these, Cyperus rotundus and Chenopodium album were the most dominant of monocot and dicot weeds respectively (Table 2).

\section{Weed population (density)}

Weed density (Population per unit area) is an important and key parameter in figuring out the impact of treatments on weed growth (Table 2). All the weed control treatments significantly reduced the population of monocot and diocot weeds over weedy check at 30 days after sowing (DAS) and harvest.

The population of monocot weeds $\mathrm{m}^{-2}$ at 30 DAS and harvest differed significantly among 
various treatments (Table 2). Minimum population of monocot weeds was recorded under treatment weeds free $\left(\mathrm{T}_{11}\right)$ which was significantly lower than rest of all other treatments. Maximum population was recorded under weedy check (T12), which was comparable to other weedicide treatments. At harvest, all weed control treatments significantly reduced the population of monocot weeds over weedy check. The minimum population was registered in weed free treatments but this was statistically at par with Sulfosulfuron + metsulfuron $\left(\mathrm{T}_{10}\right)$, Mesosulfuron + iodosulfuron $\left(\mathrm{T}_{9}\right), \quad$ Isoproturon $\left(\mathrm{T}_{8}\right)$, Sulfosulfuron $\left(\mathrm{T}_{7}\right)$, Pinoxaden + Metsulfuron $\left(\mathrm{T}_{6}\right)$ and Pinoxaden $+2,4-\mathrm{D}-\mathrm{E}\left(\mathrm{T}_{5}\right)$ treatments. The maximum population of monocot weeds was observed under weedy check. Khan et al., (2004) and Chhokar et al., (2007) recorded significant reduction in weed growth with the application of herbicides.

The population of diocot weeds at 30 DAS and harvest differed significantly due to different treatments (Table 2). The major dicot weeds observed were Chenopodium album, Anagalis arvensis, Convolvulus arvensis and Medicago hispida. At 30 DAS, the minimum population of diocot weeds was recorded under weed free $\left(\mathrm{T}_{11}\right)$ treatment which was significantly lower over rest of the treatments. The maximum population of dicot weeds was recorded in weedy check, which was comparable to all the weedicides treatments. At harvest, minimum population of diocot weeds was noted in weed free treatment $\left(\mathrm{T}_{11}\right)$ which was at par with Sulfosulfuron + metsulfuron $\left(\mathrm{T}_{10}\right)$, Isoproturon $\left(\mathrm{T}_{8}\right)$ and Pinoxaden $+2,4-\mathrm{D}-\mathrm{E}\left(\mathrm{T}_{5}\right)$, treatments. The maximum population of diocot weeds was recorded in Weedy check.

The least weed density in herbicide treatments might be due to their phytotoxicity against diverse and disruptive weed flora. These findings were in harmony with that of Khan et al., (2001). They reported that grassy and broadleaf weeds were controlled very effectively by the application of herbicides (Hassan et al., 2003; Khan et al., 2004 and Jarwar et al., 2005).

\section{Weed dry weight $\left(\mathrm{g} \mathrm{m}^{-2}\right)$}

At 30 DAS, significantly lower weed dry weight was recorded under the weed free $\left(\mathrm{T}_{11}\right)$ treatment (Table 2). Whereas, maximum dry weight was found under weedy check $\left(T_{12}\right)$. At this stage, the weed dry matter in all the weedicide treatments was comparable. At harvest, all the weed control treatments resulted significantly lower weed dry weight as compared to weedy check. The minimum weed dry weight was recorded under weed free treatment $\left(T_{11}\right)$ but was at par with Sulfosulfuron + metsulfuron $\left(\mathrm{T}_{10}\right)$, Mesosulfuron + iodosulfuron $\left(\mathrm{T}_{9}\right)$, Pinoxaden $+2,4-\mathrm{D}-\mathrm{E} \quad\left(\mathrm{T}_{5}\right), \quad$ Sulfosulfuron $\quad\left(\mathrm{T}_{7}\right)$, Isoproturon $\left(\mathrm{T}_{8}\right)$, and Pinoxaden + Metsulfuron $\left(\mathrm{T}_{6}\right)$. The maximum weed dry weight was recorded in weedy check.

The application of Sulfosulfuron + Metsulfuron, Mesosulfuron + Iodosulfuron, Isoproturon and Sulfosulfuron was effective in controlling weeds as compared to rest of the treatments as these herbicides controlled both narrow and broad leaf weeds (Pandey et al., 2006) owing to synergetic enhancement. Herbicidal combinations in general were better than sole application of herbicides in efficiency reducing the total weed dry weight.

\section{Weed control efficiency (\%)}

Maximum weed control efficiency $(92.5 \%)$ was recorded in weed free $\left(\mathrm{T}_{11}\right)$ treatment (Table 2). Under different weedicides treatments, maximum weed control efficiency was noted with Sulfosulfuron + Metsulfuron treatment $\left(\mathrm{T}_{10}\right)$ and it was followed by 
Mesosulfuron + Iodosulfuron $\quad\left(\mathrm{T}_{9}\right)$ Pinoxaden +2,4-D-E $\left(\mathrm{T}_{5}\right)$, Isoproturon $\left(\mathrm{T}_{8}\right)$, Pinoxaden + Metsulfuron $\left(\mathrm{T}_{6}\right)$ and Sulfosulfuron $\left(\mathrm{T}_{7}\right)$ treatments with 86.2, 84.6, 81.7, 81.3 and 80.9 per cent weed control efficiency, respectively. Because of better control of weeds under the herbicide mixture, weed control efficiency under these treatments was comparable to weed free. Meena et al., (2017) reported that application of tank mixed metsulfuron + sulfosulfuron mixture provided maximum per cent reduction in density and dry matter $(90.05 \&$ $95.35 \%$ ) of total weeds over unweeded control followed by mesosulfuron + iodosulfuron, clodinofop + metsulfuron and pinoxaden + metsulfuron $(88.8,88.0$ and 87.4 \& 94.7, 94.4 and $94.2 \%$ ) at 60 DAS which resulted into highest weed control efficiency (95.4, 94.7, 94.4 and 94.2\%) and proved significantly superior over rest of the herbicidal treatments. Application of herbicide alone gave poor control of weeds, therefore had lower weed control efficiency. These results are in close conformity with findings of Yadav et al., (2009) and Chopra and Chopra (2005).

\section{Weed index $(\%)$}

The weed index ranged from 2.04 to $52.04 \%$ under different treatments (Table 2). The treatment Sulfosulfuron + Metsulfuron $\left(\mathrm{T}_{10}\right)$ recorded lowest weed index. Application of Isoproturon $\left(\mathrm{T}_{8}\right)$ and Mesosulfuron + Iodosulfuron $\left(\mathrm{T}_{9}\right)$ observed 5.21 and $5.99 \%$ weed index. Whereas, weedy check showed maximum (41.23\%) weed index.

\section{Crop}

\section{Growth parameters}

Plant population ( $\mathrm{m}^{-1}$ row length) of wheat at initial and harvest stage was not significantly affected by any of the weed control treatments (Table 3). The weed control treatments significantly influenced the morphological parameters like plant height; number of tillers per plant at harvest.

At harvest, higher plant height was observed in weed free $\left(\mathrm{T}_{11}\right)$ treatment which was at par with application of Pinoxaden + Metsulfuron (T6), Sulfosulfuron + Metsulfuron $\left(\mathrm{T}_{10}\right)$, Mesosulfuron + Iodosulfuron $\left(\mathrm{T}_{9}\right)$, Isoproturon $\left(\mathrm{T}_{8}\right)$. The minimum height was noted in weedy check $\left(\mathrm{T}_{12}\right)$ treatment.

At harvest, number of tillers ranged from 8.75 to 14.57 per plant under different treatments (Table 3). At this stage, maximum number of tillers were obtained in treatment weed free $\left(\mathrm{T}_{11}\right)$ and comparable with Sulfosulfuron + metsulfuron $\left(\mathrm{T}_{10}\right), \quad$ Mesosulfuron + iodosulfuron $\left(\mathrm{T}_{9}\right), \quad$ Pinoxaden $\quad+$ Metsulfuron $\left(T_{6}\right), \quad$ Sulfosulfuron $\left(T_{7}\right)$ and Isoproturon $\left(\mathrm{T}_{8}\right)$ treatments and minimum number of tillers were observed in weedy check treatment $\left(T_{12}\right)$.

\section{Yield attributes}

The number of ear-heads per meter row length; length of ear-head, number of grains per ear-head, weight of ear head and test weight were significantly influenced due to weed control treatments (Table 4).

The maximum number of ear-heads were recorded with application of Sulfosulfuron + Metsulfuron $\left(\mathrm{T}_{10}\right)$, which was closely followed by weed free treatment $\left(T_{11}\right)$. However, the number of ear-heads per meter row length recorded in treatments, Mesosulfuron + Iodosulfuron $\left(\mathrm{T}_{9}\right)$, Isoproturon $\left(\mathrm{T}_{8}\right)$ and Pinoxaden $+2,4-\mathrm{D}-\mathrm{E}$ $\left(T_{5}\right)$ were statistically at par with each other. Whereas, minimum number of ear-heads were recorded under weedy check $\left(\mathrm{T}_{12}\right)$ treatment.

The longest ear head was observed in weed free treatment $\left(T_{11}\right)$ which was statistically at par with those received herbicides which 
controlled the grassy and broad leaf weeds, i.e. Pinoxaden +2,4-D-E $\left(T_{5}\right)$, Pinoxaden + Metsulfuron $\left(\mathrm{T}_{6}\right), \quad$ Sulfosulfuron $\quad\left(\mathrm{T}_{7}\right)$, Isoproturon $\left(\mathrm{T}_{8}\right), \quad$ Mesosulfuron + iodosulfuron $\left(\mathrm{T}_{9}\right)$ and Sulfosulfuron + metsulfuron $\left(\mathrm{T}_{10}\right)$ treatments (Table 4$)$. While, shorter ear head were observed under weedy check treatment.

Among the yield components, number of grains per spike is imperative parameter for assessment of the impact of weed management treatments on yield. Increasing the number of grains per spike will increase the weight of the spike which in turn improves the yield (Table 4). All the weed management treatments significantly boosted the number of grains per spike. Different chemical treatments had significant effects on grains per spike. The maximum number of grains and ear head weight was noted in weed free treatment $\left(\mathrm{T}_{11}\right)$ and it was significantly higher than that observed under 2,4-D-E $\left(\mathrm{T}_{1}\right)$, Metsulfuron $\left(\mathrm{T}_{2}\right)$, Pinoxaden $\left(\mathrm{T}_{3}\right)$, and Clodinafop $\left(\mathrm{T}_{4}\right)$ treatments. Under different herbicidal treatments, Pinoxaden +2,4-D$\mathrm{E}\left(\mathrm{T}_{5}\right)$, Pinoxaden + Metsulfuron $\left(\mathrm{T}_{6}\right)$, Sulfosulfuron $\left(\mathrm{T}_{7}\right)$, Isoproturon $\left(\mathrm{T}_{8}\right)$, Mesosulfuron + iodosulfuron $\left(\mathrm{T}_{9}\right)$ and Sulfosulfuron + metsulfuron $\left(\mathrm{T}_{10}\right)$ recorded statistically comparable number of grains per ear-head and their weight, while minimum values were observed under weedy check treatment $\left(\mathrm{T}_{12}\right)$.

A significant impact was noted due to different weed control treatments on 1000grain weight. The treatment $\mathrm{T}_{10}$ (Sulfosulfuron + Metsulfuron $\mathrm{T}_{10}$ ) produced the maximum test weight (42.82 g), closely followed by weed free treatment $T_{10}$. Minimum 1000 grain weight was observed in weedy check $\left(\mathrm{T}_{12}\right)$ treatment. However, treatments, Pinoxaden +2,4-D-E $\left(\mathrm{T}_{5}\right)$, Pinoxaden + Metsulfuron $\left(\mathrm{T}_{6}\right)$, Sulfosulfuron $\left(\mathrm{T}_{7}\right)$, Isoproturon $\left(\mathrm{T}_{8}\right)$, and Mesosulfuron + iodosulfuron $\left(\mathrm{T}_{9}\right)$ were at par and recorded significantly higher 1000-grain weight over 2,4-D-E $\left(\mathrm{T}_{1}\right)$, Metsulfuron $\left(\mathrm{T}_{2}\right)$, Pinoxaden $\left(\mathrm{T}_{3}\right)$, and Clodinafop $\left(\mathrm{T}_{4}\right)$ treatments (Table 4$)$.

Table.1 Details of treatments and their symbols

\begin{tabular}{|c|l|c|c|c|c|}
\hline S. No. & \multicolumn{1}{|c|}{ Treatments } & $\begin{array}{c}\text { Dose } \\
\mathbf{( g ~ h}^{-\mathbf{1}} \mathbf{)}\end{array}$ & Symbols & \multicolumn{2}{c|}{ Weed Control } \\
\hline $\mathbf{1}$ & 2,4-D-E & 500 & $\mathrm{~T}_{1}$ & & $\sqrt{ }$ \\
\hline $\mathbf{2 .}$ & Metsulfuron & 4 & $\mathrm{~T}_{2}$ & & $\sqrt{ }$ \\
\hline $\mathbf{3 .}$ & Pinoxaden & 40 & $\mathrm{~T}_{3}$ & $\sqrt{ }$ & \\
\hline $\mathbf{4 .}$ & Clodinafop & 60 & $\mathrm{~T}_{4}$ & $\sqrt{ }$ & \\
\hline $\mathbf{5 .}$ & Pinoxaden +2,4-D-E & $40+500$ & $\mathrm{~T}_{5}$ & $\sqrt{ }$ & $\sqrt{ }$ \\
\hline $\mathbf{6 .}$ & Pinoxaden + Metsulfuron & $40+4$ & $\mathrm{~T}_{6}$ & $\sqrt{ }$ & $\sqrt{ }$ \\
\hline $\mathbf{7 .}$ & Sulfosulfuron & 25 & $\mathrm{~T}_{7}$ & $\sqrt{ }$ & $\sqrt{ }$ \\
\hline $\mathbf{8 .}$ & Isoproturon & 1000 & $\mathrm{~T}_{8}$ & $\sqrt{ }$ & $\sqrt{ }$ \\
\hline $\mathbf{9 .}$ & Atlantis (Mesosulfuron + iodosulfuron) & $12+2.4$ & $\mathrm{~T}_{9}$ & $\sqrt{ }$ & $\sqrt{ }$ \\
\hline $\mathbf{1 0 .}$ & Total (Sulfosulfuron + metsulfuron) & $30+2$ & $\mathrm{~T}_{10}$ & $\sqrt{ }$ & $\sqrt{ }$ \\
\hline $\mathbf{1 1}$. & Weed free (Two HW at 30 \& 50 DAYS) & & $\mathrm{T}_{11}$ & & \\
\hline $\mathbf{1 2 .}$ & Weedy check & & $\mathrm{T}_{12}$ & & \\
\hline
\end{tabular}


Table.2 Effect of different treatments on population of monocot and dicot weeds per square meter in wheat

\begin{tabular}{|c|c|c|c|c|c|c|c|c|}
\hline \multirow{4}{*}{\begin{tabular}{|l} 
\\
Treatments
\end{tabular}} & \multirow{2}{*}{\multicolumn{4}{|c|}{ Weed population, number $\mathrm{m}^{-2}$}} & \multirow{2}{*}{\multicolumn{2}{|c|}{$\begin{array}{l}\text { Weed dry weight, } \\
\mathrm{g} \mathrm{m}^{-2}\end{array}$}} & \multirow{4}{*}{$\begin{array}{c}\text { Weed } \\
\text { control } \\
\text { efficiency, } \\
\% \\
\text { Av. }(60,90 \\
\text { DAS \& } \\
\text { harvest) }\end{array}$} & \multirow{4}{*}{$\begin{array}{c}\text { Weed } \\
\text { index, } \\
\%\end{array}$} \\
\hline & & & & & & & & \\
\hline & \multicolumn{2}{|c|}{ Monocot } & \multicolumn{2}{|c|}{ Dicot } & & & & \\
\hline & 30 & Maturity & 30 & Maturity & 30 & Harvest & & \\
\hline 2,4-D-E & $\begin{array}{c}9.33 \\
(3.05)\end{array}$ & $\begin{array}{c}9.67 \\
(3.11)\end{array}$ & $\begin{array}{l}10.33 \\
(3.21)\end{array}$ & $\begin{array}{l}5.00 \\
(2.24)\end{array}$ & 14.96 & 4.0 & 75.7 & 19.03 \\
\hline Metsulfuron & $\begin{array}{c}9.00 \\
(1.63)\end{array}$ & $\begin{array}{c}8.33 \\
(1.79)\end{array}$ & $\begin{array}{c}9.67 \\
(3.11)\end{array}$ & $\begin{array}{c}5.33 \\
(2.31)\end{array}$ & 15.54 & 3.16 & 77.8 & 22.70 \\
\hline Pinoxaden & $\begin{array}{c}9.67 \\
(3.11)\end{array}$ & $\begin{array}{c}5.33 \\
(2.27)\end{array}$ & $\begin{array}{l}10.00 \\
(3.16)\end{array}$ & $\begin{array}{c}8.00 \\
(2.83)\end{array}$ & 14.44 & 3.44 & 77.8 & 19.23 \\
\hline Clodinafop & $\begin{array}{l}10.00 \\
(3.16)\end{array}$ & $\begin{array}{c}2.67 \\
(1.57)\end{array}$ & $\begin{array}{l}10.67 \\
(3.27)\end{array}$ & $\begin{array}{c}9.33 \\
(3.05)\end{array}$ & 14.92 & 4.12 & 77.8 & 19.12 \\
\hline $\begin{array}{l}\text { Pinoxaden +2,4- } \\
\text { D-E }\end{array}$ & $\begin{array}{c}9.33 \\
(3.05)\end{array}$ & $\begin{array}{c}3.00 \\
(1.73)\end{array}$ & $\begin{array}{c}9.67 \\
(3.11)\end{array}$ & $\begin{array}{c}3.00 \\
(1.73)\end{array}$ & 13.88 & 1.88 & 86.1 & 9.81 \\
\hline $\begin{array}{l}\text { Pinoxaden + } \\
\text { Metsulfuron }\end{array}$ & $\begin{array}{c}9.67 \\
(3.11)\end{array}$ & $\begin{array}{c}2.33 \\
(1.53)\end{array}$ & $\begin{array}{l}10.00 \\
(3.16)\end{array}$ & $\begin{array}{c}4.33 \\
(2.08)\end{array}$ & 14.28 & 2.44 & 82.4 & 7.81 \\
\hline Sulfosulfuron & $\begin{array}{l}10.67 \\
(3.27)\end{array}$ & $\begin{array}{c}2.33 \\
(1.53) \\
\end{array}$ & $\begin{array}{c}9.67 \\
(3.11) \\
\end{array}$ & $\begin{array}{c}3.67 \\
(1.92)\end{array}$ & 15.12 & 2.08 & 83.3 & 7.18 \\
\hline Isoproturon & $\begin{array}{l}11.00 \\
(3.29)\end{array}$ & $\begin{array}{c}3.33 \\
(1.82)\end{array}$ & $\begin{array}{c}9.33 \\
(3.05)\end{array}$ & $\begin{array}{c}3.00 \\
(1.73)\end{array}$ & 13.65 & 2.16 & 81.8 & 5.21 \\
\hline $\begin{array}{l}\text { Atlantis } \\
\text { (Mesosulfuron + } \\
\text { iodosulfuron) }\end{array}$ & $\begin{array}{c}9.00 \\
(3.00)\end{array}$ & $\begin{array}{c}2.00 \\
(1.41)\end{array}$ & $\begin{array}{c}9.67 \\
(3.11)\end{array}$ & $\begin{array}{c}4.00 \\
(2.00)\end{array}$ & 13.55 & 1.44 & 87.7 & 5.99 \\
\hline $\begin{array}{l}\text { Total } \\
\text { (Sulfosulfuron + } \\
\text { metsulfuron) }\end{array}$ & $\begin{array}{c}9.33 \\
(3.05)\end{array}$ & $\begin{array}{c}1.33 \\
(1.15)\end{array}$ & $\begin{array}{c}9.33 \\
(3.05)\end{array}$ & $\begin{array}{c}1.33 \\
(1.15)\end{array}$ & 12.88 & 1.26 & 89.5 & 2.04 \\
\hline $\begin{array}{l}\text { Weed free (Two } \\
\text { HW at } 30 \& 50 \\
\text { DAS) }\end{array}$ & $\begin{array}{c}2.00 \\
(1.41)\end{array}$ & $\begin{array}{c}1.00 \\
(1.00)\end{array}$ & $\begin{array}{c}1.33 \\
(1.15)\end{array}$ & $\begin{array}{c}1.00 \\
(1.00)\end{array}$ & 1.14 & 1.08 & 92.5 & 0.00 \\
\hline Weedy check & $\begin{array}{r}11.33 \\
(3.37) \\
\end{array}$ & $\begin{array}{l}15.00 \\
(3.87)\end{array}$ & $\begin{array}{l}11.33 \\
(3.37)\end{array}$ & $\begin{array}{l}17.67 \\
(4.20)\end{array}$ & 19.42 & 23.04 & 0.0 & 52.04 \\
\hline S.Em. \pm & 0.82 & 0.75 & 1.05 & 0.73 & 0.84 & 0.70 & & \\
\hline C.D. at $5 \%$ & 2.42 & 2.20 & 3.10 & 2.16 & 2.48 & 2.07 & & \\
\hline
\end{tabular}

Figure in parenthesis indicate the transformation values. 
Table.3 Effect of different treatments on the plant population meter $^{-2}$ row length of wheat

\begin{tabular}{|l|l|c|c|c|c|}
\hline Tr. No. & Treatments & \multicolumn{2}{|c|}{$\begin{array}{c}\text { Plant population } \\
\text { Per meter row length }\end{array}$} & $\begin{array}{c}\text { Plant } \\
\text { height at } \\
\text { harvest, } \\
\text { cm }\end{array}$ & $\begin{array}{c}\text { Tillers per } \\
\text { culm at } \\
\text { harvest, } \\
\text { number }\end{array}$ \\
\hline $\mathbf{T}_{\mathbf{1}}$ & 2,4-D-E & 30 DAS & Harvest & 88.24 & 11.19 \\
\hline $\mathbf{T}_{\mathbf{2}}$ & Metsulfuron & 18.16 & 16.79 & 88.86 & 11.62 \\
\hline $\mathbf{T}_{\mathbf{3}}$ & Pinoxaden & 18.30 & 16.62 & 87.24 & 11.44 \\
\hline $\mathbf{T}_{\mathbf{4}}$ & Clodinafop & 17.50 & 16.21 & 90.06 & 11.16 \\
\hline $\mathbf{T}_{\mathbf{5}}$ & Pinoxaden+2,4-D-E & 18.66 & 16.62 & 95.00 & 12.34 \\
\hline $\mathbf{T}_{\mathbf{6}}$ & Pinoxaden + Metsulfuron & 17.45 & 16.16 & 100.61 & 13.64 \\
\hline $\mathbf{T}_{\mathbf{7}}$ & Sulfosulfuron & 17.89 & 17.18 & 96.15 & 13.43 \\
\hline $\mathbf{T}_{\mathbf{8}}$ & Isoproturon & 18.22 & 16.87 & 98.65 & 13.32 \\
\hline $\mathbf{T}_{\mathbf{9}}$ & Atlantis (Mesosulfuron + & 18.11 & 16.51 & 99.16 & 14.02 \\
\hline & iodosulfuron) & 17.94 & 16.90 & 99.85 & 14.23 \\
\hline $\mathbf{T}_{\mathbf{1 0}}$ & Total (Sulfosulfuron + metsulfuron) & 18.00 & 16.97 & 104.60 & 14.57 \\
\hline $\mathbf{T}_{\mathbf{1 1}}$ & Weed free (Two HW at 30 \& 50 & 18.11 & 16.97 & 79.24 & 8.75 \\
\hline & DAS) & & & & \\
\hline $\mathbf{T}_{\mathbf{1 2}}$ & Weedy check & 18.33 & 16.31 & 2.46 & 0.54 \\
\hline & S.Em. \pm & 0.32 & 0.31 & 7.22 & 1.60 \\
\hline & C.D. at 5\% & NS & NS & 88.24 & 11.19 \\
\hline
\end{tabular}

Table.4 Yield attributing character of wheat as influenced by weed control treatments

\begin{tabular}{|l|l|c|c|c|c|c|}
\hline $\begin{array}{l}\text { Tr. } \\
\text { No. }\end{array}$ & Treatments & $\begin{array}{c}\text { Number of } \\
\text { ear-heads } \\
\text { /m row } \\
\text { length }\end{array}$ & $\begin{array}{l}\text { Length of } \\
\text { earhead } \\
\text { (cm) }\end{array}$ & $\begin{array}{l}\text { Number } \\
\text { of grains } \\
\text { earhead }\end{array}$ & $\begin{array}{l}\text { Weight } \\
\text { of ear } \\
\text { head (g) }\end{array}$ & $\begin{array}{c}\text { Test } \\
\text { weight } \\
\text { (g) }\end{array}$ \\
\hline $\mathbf{T}_{\mathbf{1}}$ & 2,4-D-E & 47.61 & 7.47 & 36.33 & 8.62 & 38.98 \\
\hline $\mathbf{T}_{\mathbf{2}}$ & Metsulfuron & 48.91 & 7.42 & 36.18 & 8.56 & 38.75 \\
\hline $\mathbf{T}_{\mathbf{3}}$ & Pinoxaden & 49.77 & 7.44 & 36.22 & 8.59 & 38.85 \\
\hline $\mathbf{T}_{\mathbf{4}}$ & Clodinafop & 49.64 & 7.45 & 36.30 & 8.60 & 38.91 \\
\hline $\mathbf{T}_{\mathbf{5}}$ & Pinoxaden +2,4-D-E & 55.58 & 8.31 & 40.45 & 9.59 & 42.06 \\
\hline $\mathbf{T}_{\mathbf{6}}$ & Pinoxaden + Metsulfuron & 52.59 & 8.50 & 41.34 & 9.64 & 42.02 \\
\hline $\mathbf{T}_{\mathbf{7}}$ & Sulfosulfuron & 52.29 & 8.55 & 41.60 & 9.73 & 41.63 \\
\hline $\mathbf{T}_{\mathbf{8}}$ & Isoproturon & 55.26 & 8.74 & 42.51 & 9.96 & 41.61 \\
\hline $\mathbf{T}_{\mathbf{9}}$ & Atlantis (Mesosulfuron + iodosulfuron) & 55.87 & 8.66 & 42.15 & 9.90 & 42.22 \\
\hline $\mathbf{T}_{\mathbf{1 0}}$ & Total (Sulfosulfuron + metsulfuron) & 58.58 & 8.78 & 42.70 & 9.99 & 42.82 \\
\hline $\mathbf{T}_{\mathbf{1 1}}$ & Weed free (Two HW at 30 \& 50 DAS) & 57.96 & 8.88 & 43.22 & 10.02 & 42.70 \\
\hline $\mathbf{T}_{\mathbf{1 2}}$ & Weedy check & 34.32 & 6.76 & 32.84 & 7.80 & 33.94 \\
\hline & S.Em. (E) & $\mathbf{1 . 5 0}$ & $\mathbf{0 . 2 1}$ & $\mathbf{1 . 0 1}$ & $\mathbf{0 . 2 0}$ & $\mathbf{0 . 8 6}$ \\
\hline & C.D. at 5\% & $\mathbf{4 . 3 9}$ & $\mathbf{0 . 6 1}$ & $\mathbf{2 . 9 5}$ & $\mathbf{0 . 5 9}$ & $\mathbf{2 . 5 3}$ \\
\hline
\end{tabular}


Table.5 Effect of different treatments on grain, straw and biological yield $\left(\mathrm{kg} \mathrm{ha}^{-1}\right)$ and harvest index (\%) of wheat

\begin{tabular}{|l|l|c|c|c|c|}
\hline $\begin{array}{c}\text { Tr. } \\
\text { No. }\end{array}$ & Treatments & $\begin{array}{c}\text { Grain } \\
\text { yield } \\
\left(\mathbf{K g ~ h a}^{-1}\right)\end{array}$ & $\begin{array}{c}\text { Straw } \\
\text { yield } \\
\left(\mathbf{k g ~ h a}^{-\mathbf{1}}\right)\end{array}$ & $\begin{array}{c}\text { Biological } \\
\text { yield } \\
\left(\mathbf{k g ~ h a}^{-\mathbf{1}} \mathbf{)}\right.\end{array}$ & $\begin{array}{c}\text { Harvest } \\
\text { index } \\
\mathbf{( \% )}\end{array}$ \\
\hline $\mathbf{T}_{\mathbf{1}}$ & 2,4-D-E & 3731 & 4686 & 8417 & 44.34 \\
\hline $\mathbf{T}_{\mathbf{2}}$ & Metsulfuron & 3562 & 4613 & 8175 & 43.55 \\
\hline $\mathbf{T}_{\mathbf{3}}$ & Pinoxaden & 3722 & 4631 & 8352 & 44.57 \\
\hline $\mathbf{T}_{\mathbf{4}}$ & Clodinafop & 3727 & 4847 & 8574 & 43.47 \\
\hline $\mathbf{T}_{\mathbf{5}}$ & Pinoxaden +2,4-D-E & 4156 & 5137 & 9293 & 44.72 \\
\hline $\mathbf{T}_{\mathbf{6}}$ & Pinoxaden + Metsulfuron & 4248 & 5196 & 9445 & 44.99 \\
\hline $\mathbf{T}_{\mathbf{7}}$ & Sulfosulfuron & 4277 & 5250 & 9527 & 44.87 \\
\hline $\mathbf{T}_{\mathbf{8}}$ & Isoproturon & 4368 & 5153 & 9521 & 45.88 \\
\hline $\mathbf{T}_{\mathbf{9}}$ & Atlantis (Mesosulfuron + iodosulfuron) & 4332 & 5052 & 9384 & 46.16 \\
\hline $\mathbf{T}_{\mathbf{1 0}}$ & Total (Sulfosulfuron + metsulfuron) & 4514 & 5141 & 9655 & 46.75 \\
\hline $\mathbf{T}_{\mathbf{1 1}}$ & Weed free (Two HW at 30 \& 50 DAS) & 4608 & 5187 & 9795 & 47.02 \\
\hline $\mathbf{T}_{\mathbf{1 2}}$ & Weedy check & 2210 & 3198 & 5408 & 40.80 \\
\hline & S.Em. \pm & $\mathbf{1 2 0}$ & $\mathbf{1 2 1}$ & $\mathbf{2 2 1}$ & $\mathbf{0 . 6 9}$ \\
\hline & C.D. at 5\% & $\mathbf{3 5 1}$ & $\mathbf{3 5 4}$ & $\mathbf{6 4 7}$ & $\mathbf{2 . 0 1}$ \\
\hline
\end{tabular}

\section{Yield}

Significant effect on wheat grain yield was noticed among various herbicide treatments (Table 5). The maximum grain yield was obtained in weed free treatment which was statistically at par with Sulfosulfuron + Metsulfuron $\left(\mathrm{T}_{10}\right)$ herbicide treatnent. The grain yield observed with application of Isoproturon $\left(\mathrm{T}_{8}\right), \quad$ Mesosulfuron + Iodosulfuron( $\mathrm{T}_{9}$ ) and Sulfosulfuron (T7) were also statistically comparable to each other. Due to maximum infestation of weeds, the lowest wheat grain yield was recorded in the weedy check plots.

The higher grain yield in herbicides treated plots is due to better control of grassy and broad-leaved weeds thus the crop was able to utilize the available resources more efficiently. Similar findings were reported by earlier researchers (Hassan et al., 2003; Tunio et al., 2004; Hesammi et al., 2010; Mahmood et al., 2012; Shahzad et al., 2012; Hussain et al., 2013; Singh et al., 2013).

All weed control treatments significantly increased the straw yield over weedy check (Table 5). Highest straw yield was recorded in Sulfosulfuron treated plot which was statistically at par with that of weed free $\left(\mathrm{T}_{11}\right)$, Sulfosulfuron+ Metsulfuron $\left(\mathrm{T}_{10}\right)$, Mesosulfuron + Iodosulfuron $\left(\mathrm{T}_{9}\right)$, Isoproturon (T8), Pinoxaden + Metsulfuron $\left(\mathrm{T}_{6}\right)$ and Pinoxaden $+2,4-\mathrm{D}-\mathrm{E}\left(\mathrm{T}_{5}\right)$ treated plots. The lower straw yield was recorded under weedy check which was significantly inferior to that under rest of the treatments. The highest straw yield could be owing to the better management of mono and dicot weeds by herbicidal treatments and thus the crop was capable to make use of the available resources more proficiently. The similar findings were reported previously by researchers (Khan et al., 2003; Singh et al., 2013). 
The biological yield ranged from 5408 to $9795 \mathrm{~kg} \mathrm{ha}^{-1}$ under different weed control treatments. Significantly greater biological yield was noted in treatment weed free $\left(\mathrm{T}_{11}\right)$ which was statistically at par with Sulfosulfuron + metsulfuron $\left(\mathrm{T}_{10}\right)$, Mesosulfuron + iodosulfuron $\left(\mathrm{T}_{9}\right)$, Isoproturon $\left(\mathrm{T}_{8}\right), \quad$ Sulfosulfuron $\left(\mathrm{T}_{7}\right)$, Pinoxaden + Metsulfuron $\left(\mathrm{T}_{6}\right)$ and Pinoxaden +2,4-D-E $\left(\mathrm{T}_{5}\right)$ treatments (Table 5). Significantly lower biological yield was recorded by the treatment weedy check $\left(\mathrm{T}_{12}\right)$ which was found to be statistically inferior to rest of the treatments.

The harvest index was in the range of 40.80 to $47.02 \%$ under different weed control treatments. Maximum harvest index (47.02 $\%)$ was observed under weed free $\left(\mathrm{T}_{11}\right)$ treatment which was comparable with Sulfosulfuron + metsulfuron $\left(\mathrm{T}_{10}\right)$, Mesosulfuron + iodosulfuron $\left(\mathrm{T}_{9}\right)$, Isoproturon $\left(\mathrm{T}_{8}\right)$ treatments. Minimum harvest index $(40.80 \%)$ was observed under weedy check treatment.

From present study, it can be concluded that Sulfosulfuron + metsulfuron $\left(30+2 \mathrm{~g} \mathrm{ha}^{-1}\right)$ or isoproturon $\left(1000 \mathrm{~g} \mathrm{ha}^{-1}\right)$ are most remunerative and effective herbicides for weed management in irrigated wheat under sandy loam soils of Northern Madhya Pradesh.

\section{References}

Chhokar, R. S., Sharma, R. K., Jat, G. R., Pundir, A. K. and Gathala, M. K. (2007) Effect of tillage and herbicides on weeds and productivity of wheat under rice-wheat growing system. Crop Protection 26: 1689-1696.

Chopra, N. and Chopra, N. K. (2005) Bioefficacy of fenoxaprop, clodinafop, metribuzin alone and in combinations against weeds in wheat and their residual effect on succeeding crops. Indian Journal of Weed Science 37 (3\&4): 163-166.

Damalas, C. A. and Eleftherohorinos, I. G. (2001) Dicamba and atrazine antagonism on sulfonylurea herbicides used for Johnsongrass (Sorghum halepense) control in corn

Damalas, C. A. (2004). Herbicide tank mixtures: Common interactions. International Journal of Agriculture Biology, 6: 209-212.

Dixit, A. and Bhan, V. M. (1997) Efficiency of metribuzin for controlling weeds in wheat. Ann. Rep. NRC Weed Sci. pp. 23.

Hassan, G., Faiz, B., Marwat, K. B. and Khan, M. (2003) Effects of planting method and tank mixed herbicides on controlling grassy and broadleaf weeds and their effect on wheat cv Fakhr-eSarhad. Pak. J. Weed Sci. Res., 9: 1-11.

Jarwar, A. D., Arain, M. A. and Rajput L. S. (2005) Chemical weed control in wheat. Pak. J. Weed Sci. Res., 11: 11-15.

Khan, I., Hassan, G., Khan, M. A. and Khan M. I. (2004) Efficacy of some new herbicides on both grassy and broad leaf weeds in wheat. II. Pak. J. Weed Sci. Res., 10: 33-38.

Khan, I. U., Muhammad, Z., Hassan, G. and Marwat, K. B. (2001) Efficacy of different herbicides for controlling weeds in wheat crop.I. Response of agronomic and morphological traits in wheat variety Ghaznavi. Sci. Khyber, 14: 51-57.

Khan, N., Hassan, G., Khan, M. A. and Khan, I. (2003) Efficacy of different herbicides for controlling weeds in wheat crop at different times of application-I. Asian J. Plant Sci., 2: 305-309.

Kumara, S., Ranaa, S.S., Badiyalaa, D., Suresh Kumara, Sharma, N. (2019). Bioefficacy of post-emergence 
herbicide Prioxofop-Propanyl (Markclodina) against weeds in wheat. Journal of Research in Weed Science 2 (2019) 141-148

Malik, R. K. and Singh, S. (1995) Little seed canarygrass (Phalaris minor Retz.) resistance to isoproturon in India. Weed Technology 9: 419-425.

Mathiassen, S. K. and Kudsk, P. (1998) Influence of broadleaved weed herbicides on the activity of fenoxaprop-p-ethyl. Weed Research 38: 283-289.

Meena, V., Kaushik, M.K., Meena, S.K., Bhimwal, J.P. and Chouhan, B.S. (2017). Influence of pre and post emergence herbicide application on weed growth and nutrient removal in wheat (Triticum aestivum L.). Journal of Pharmacognosy and Phytochemistry. 6(6): 2413-2418

Pandey, I. B., Dwivedi, D. K. and Pandey, R. K. (2006) Efficacy of herbicides and fertilizer management on weed dynamics of wheat. National Symposium on Conservation
Agriculture and Environment, October 26-28, at BHU, Varanasi, India, pp. 336-337.

Panse, V.G. and Sukhatme, P.V. (1989). Statistical methods for agriculture of workers. $5^{\text {th }}$ Ed. ICAR, New Delhi.

Singh, G. and Singh, M. (2002) Bio-efficacy of metsulfuron methyl in combination with isoproturon for control of grassy and non grassy weeds in wheat. Indian J. Weed Sc. 34: 9-12.

Singh, G. and Singh, M. (2002) Bio-efficacy of metsulfuron methyl in combination with isoproturon for control of grassy and non grassy weeds in wheat. Indian J. Weed Sc. 34: 9-12.

Singh, R. K., Singh, S. R. K. and Gautam, U. S. (2013) Weed Control Efficiency of herbicides in Irrigated Wheat (Triticum aestivum). Indian Res. J. Ext. Edu., 13: 126-128.

Yadav, D. B., Punia, S. S., Yadav A. and Lal R. (2009) Compatibility of sulfosulfuron with carfentrazone-ethyl for the control of complex weeds flora in wheat.

\section{How to cite this article:}

Deepesh Jaiswal, M. Devender Reddy, Girish Pandey and Anuj Kumar. 2020. Evaluation of Herbicides for Control of Weeds in Wheat (Triticum aestivum L.). Int.J.Curr.Microbiol.App.Sci. 9(05): 1157-1167. doi: https://doi.org/10.20546/ijcmas.2020.905.127 